\title{
The perception of semantic relations in pictures
}

\author{
NORMAN S. SEGALOWITZ \\ Concordia University, Montreal, Quebec H3G IM8, Canada
}

\begin{abstract}
Three picture processing experiments are reported that present evidence of an "agent advantage effect," that is, faster processing of the performer of the action depicted (the agent of the action) than of the element acted upon (the patient). Experiment 1 demonstrated the effect in a paradigm in which reaction time to manually indicate the location of a target (agent or patient) was faster for agent targets. Experiment 2 demonstrated that the critical time required for subjects to encode the agent was shorter than the time required for encoding patients. Experiment 3 again demonstrated the agent advantage effect in a directed attention paradigm. Together, the results of the experiments indicated that subjects were able to encode agent information faster than patient information. The results also indicated that subjects did not adopt a strategy whereby encoding of patient information was necessarily dependent upon encoding agent information.
\end{abstract}

Several studies have suggested that in the perception of pictured events there is a basic difference in the ways information about the semantic relations agent (the performer of an action) and patient (the person or thing acted upon) are processed. For example, Olson and Filby (1972) conducted a series of experiments in which subjects answered questions such as "What hit?" and "What was hit?" about a line drawing showing a car (agent) bumping a truck (patient). Their results indicated faster responding to questions about the agent, leading them to conclude that "it is harder to 'read' the picture in terms of the receiver of the action even when $S$ is set to look for it" (p. 379). Glucksberg, Trabasso, and Wald (1973, p.361) and Wannemacher (1976, p. 52) also suggest from their picture-sentence verification studies that the agent may precede the patient in the mental representation of the agent-patient relations in pictures. Together, these studies point to a possible difference in the way agents and patients are processed in picture perception.

The possibility of an agent-patient processing difference in the perception of visual material is of considerable interest because it may be relevant to the question of what is responsible for the well documented agentpatient differences in language. For example, some linguists have argued for a universal grammatical constraint giving agents priority over patients in being realized as the grammatical subject of a sentence (Keehan, 1976). Also, an agent-then-patient order of mention is more common in the world's languages than is the reverse order, perhaps because of the nearly

This research was supported by Grant A0306 from the National Research Council of Canada. The author wishes to thank the editor and two anonymous reviewers for their constructive comments on an earlier version of this manuscript. Requests for reprints should be sent to Norman Segalowitz, Psychology Department, Concordia University, 1455 de Maisonneuve Boulevard, West, Montreal, Quebec H3G 1M8, Canada. universal subject-before-object order-of-mention constraint (Greenberg, 1963; Pullum, 1977; Steele, 1978). Researchers of language development have noted that children in English language environments master active sentences (with agent as subject, agent mentioned before patient) before passive sentences (e.g., Baldie, 1976). Children are reported to often interpret the first noun of a sentence as the agent and the second as the patient when other clues to the correct interpretation are absent (Bever, 1970; Segalowitz \& Galang, 1978; Slobin, 1973; Strohner \& Nelson, 1974).

The present experiments explore some aspects of agent-patient differences in a picture perception paradigm. Experiment 1 demonstrates a difference favoring agents in a simple identification task, referred to henceforth as the agent advantage effect. This effect is further examined in Experiment 2 to determine whether it reflects a difference at the level of information encoding or a difference in the organization of that information at a later stage. Experiment 3 looks further at the possibility that the agent advantage effect reflects perceptual processing.

\section{EXPERIMENT 1}

This experiment investigated the relative ease with which viewers could locate the agent or patient in a picture. Subjects viewed still line drawings of an agent acting upon a patient and indicated manually the location of the agent or patient. The paradigm used in this experiment permitted control over two factors that may have contributed artifactually to the effect in previously reported studies. One factor is the use of sentences to probe perception of the agent or patient either directly (Olson \& Filby, 1972) or indirectly by having the subject look for a picture-sentence mismatch (Glucksberg et al., 1973; Wannemacher, 1974). The use of sentences may "set" the subject to process in a verbal mode and, 
therefore, to construct representations of the picture stimuli to be maximally compatible with representations of the sentences. Since one could argue that English syntax "favors" the agent over the patient for earlier mention in sentences (e.g., Keenan, 1976), subjects may have attempted to represent the relational information in the picture in a manner congruent with a verbal representation of the same relations (cf. Clark, Carpenter, \& Just, 1973). The present study attempts to remove this factor by using only picture stimuli and nonverbal (i.e., manual) responses.

The second factor concerns the presence of physical cues in the pictures not relevant to the definition of the agent-patient relation but nevertheless correlated with “agentness." For example, in Olson and Filby (1972), the agent vehicle had wavy line contours for the portion bumping the patient vehicle whereas the patient vehicle had only nonwavy line contours. Also, the agent vehicle was always depicted above the patient on a slope (i.e., coming downhill). It is possible, therefore, that subjects responded to this information instead to of the agentness or patientness of the target vehicles.

In the present experiment, subjects were given brief presentations of pictures showing two fish, with the leftmost, the rightmost, or neither fish biting the other (see Figures 1a-1c). In one block of trials, subjects indicated the side on which the agent fish was located by pressing the left or right reaction time panel, or both panels when no target appeared. In a second block of trials, subjects indicated the side on which the patient fish was located. An agent advantage effect would be revealed by faster reaction times to locate the agent than to locate the patient.

The inclusion of the no-target trials was intended to encourage subjects to attend closely to the critical parts of the picture and to avoid looking for other cues, such as the orientation of the fish, which they might think were correlated with agentness or patientness.

As another control to test whether subjects were coding the pictures in terms of some physical characteristics rather than in terms of the agent-patient relations, a second condition was devised using stimuli resembling the fish but lacking agent-patient relations. These were letter W shapes (Figure 1d) drawn to preserve the essential contours defining the biting action in the fish pictures. Subjects were given spatial relation definitions that identified either the agent-like $W$ or the patient-like $W$ without creating a set to look for an agent-patient relation. The task for the spatial relation control group was to locate the target W element. An advantage effect obtained with the agent-like Ws would question the need for appealing to agent-patient relations to explain the agent advantage effect with the fish stimuli. This is because the $\mathrm{W}$ pictures contain all the relevant spatial configurational features present in the fish pictures, but not the agent-patient relation. An interaction effect, on the other hand, indicating faster reaction times to locate the agent fish but no reaction time advantage to locate the agent-like Ws, would support the conclusion that the advantage effect with the fish is a function of the agent-patient relations perceived in the pictures.

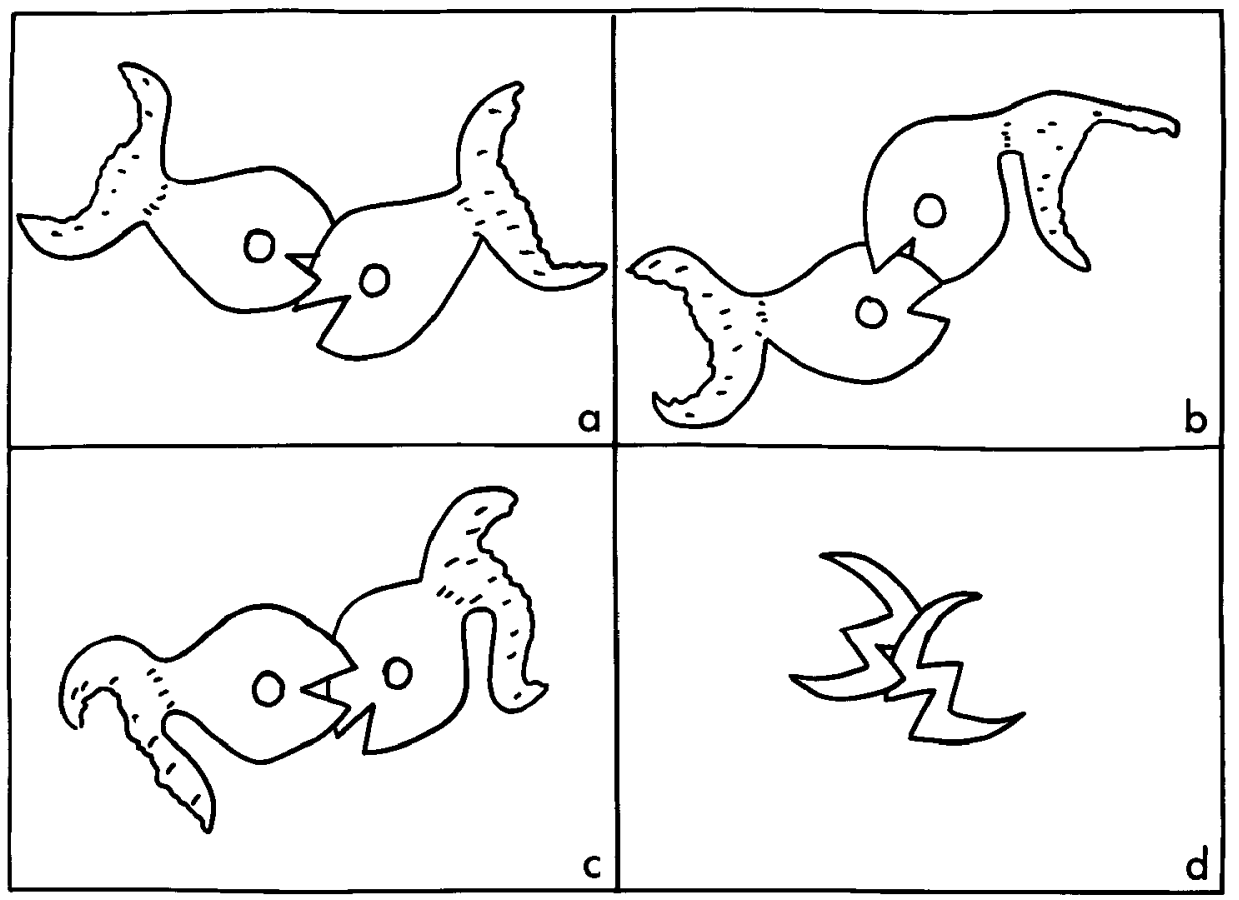

Figure 1. Sample stimuli from Experiment 1. 


\section{Method}

Subjects. Subjects were 40 paid Concordia University students with normal or corrected-to-normal vision.

Materials. The stimuli for the experimental condition were line drawings of fish depicting either a biting action (Figures 1a and $1 \mathrm{~b}$ ) or no action (Figure 1c). One-third of the basic deck of 36 stimulus cards showed the leftmost fish biting the other, one-third showed the rightmost fish biting, and one-third were of the no-action type.

To discourage subjects from attending to specific cues in the pictures other than the contours defining the biting action, the fish were drawn with varying lengths of tails and with bodies in varying orientation (sometimes biting from above, below, and at other angles). In half the action stimuli, the agent fish was drawn in red outline and the patient in black, and in half, the colors were reversed. The no-action stimuli were based on tracings from the action stimuli with the appropriate modification in the center to remove the biting action content.

The stimuli for the control condition were line drawings of two partially overlapping letter Ws. These Ws were formed by copying the biting sites in the fish pictures and replacing the tails with new contours to complete the outline of a solid letter $\mathrm{W}$ (Figure 1d was derived from Figure 1a in this manner). In this way, the control stimuli conserved the relevant physical features of the fish pictures while making it possible to steer the subject away from perceiving them in terms of an action relation.

All stimuli were drawn on $3 \times 5$ in. white cards and presented in a four-channel Gerbrands tachistoscope.

Design. This study used a 2 by 2 design, with the betweensubjects factor of relation (action vs. spatial relation) and the within-subjects factor of target (agent vs. patient). Half the subjects performed the agent task first, and half, the patient task first.

For each task (agent, patient), there were 72 test presentations, of which 48 actually contained the target. Stimulus cards were arranged in a pseudorandom manner, with the constraint that no particular response (press left, right, both) be required more than three consecutive times.

Procedure. Each subject participated in two blocks of trials. In the action relation condition, subjects performed a block of agent-search trials and a block of patient-search trials with the fish pictures described above. The subjects' task in the agentsearch block of trials was to indicate by pressing the appropriate reaction time panel which fish was "giving a bite." One-third of the time, the target was the fish on the left side of the picture (as in Figure 1a); one-third of the time, it was on the right side (Figure 1b). One-third of the time, neither fish qualified as target (as in Figure 1c), in response to which subjects pressed both panels. The subjects' task in the patient-search block of trials was to indicate which fish was "receiving a bite."

Stimulus presentation was as follows. Subjects were given a verbal "ready" signal at the onset of each trial, after which they viewed a fixation dot for 1,500 msec. Next, they saw a stimulus picture for $125 \mathrm{msec}$, followed by a 100 -msec red and black random line pattern mask. At the beginning of each block (agent, patient search), subjects were trained with a few examples presented first at $500 \mathrm{msec}$ and then gradually reduced to $125 \mathrm{msec}$. After 10 successful consecutive examples at $125 \mathrm{msec}$, subjects were told the practice work was over and the test trials would begin. They were then presented another 77 trials. The first five were buffer trials and were not included in the analyses. The remaining 72 trials represent two runs through a basic sequence of 36 cards, once with the cards in one orientation and then once rotated $180 \mathrm{deg}$. Subjects' reaction times to indicate the location of the target (or its absence) were recorded from stimulus onset.

In the spatial relation control condition, subjects were presented pictures of two partially overlapping letter Ws. In the agent block of trials, subjects had to identify the $W$ that had a point (one of the two angles at the base of the W) on top of an arm of the other (e.g., the leftmost $W$ in Figure 1d). In the patient block, subjects had to identify the $W$ that had an arm on top of a point of the other (rightmost $W$ in Figure 1d). In each block, the target $\mathrm{W}$ occurred one-third of the time on the left side of the pair and one-third of the time on the right side, and one-third of the time, neither W qualified as target.

Subjects were introduced to the task with warm-up trials using pictures of other angular letters. This was done in order to encourage subjects to see the stimuli as letters rather than as elements of an action relation. In all other respects, the procedural details of the control tasks were similar to those of the experimental condition.

Subjects were encouraged to respond as quickly as possible without sacrificing accuracy.

\section{Results}

The data were subjected to a 2 by 2 analysis of variance, with the factors being relation (action vs. spatial) and target (agent vs. patient). Only the data from trials actually containing a target were analyzed. The result of principal interest is the significant Relation by Target interaction $[\mathrm{F}(1,38)=48.46, \mathrm{MSe}=4,449, \mathrm{p}<.001]$, showing an agent advantage effect for the group receiving the action relation stimuli (514 vs. $619 \mathrm{msec}$, $\mathrm{p}<.01)$ and, if anything, the reverse for the spatial relation stimulus group ( 859 vs. $756 \mathrm{msec}, \mathrm{p}<.01)$. (All post hoc comparisons reported here are by the Newman-Keuls procedure.) It was also found that reaction times were faster in the action relation condition than in the spatial relation condition (566 vs. $807 \mathrm{msec}$ ) $[F(1,38)=24.79, \quad M S e=46,860, p<.001]$. Finally, when the data were reanalyzed to include order of task (agent task first vs. patient task first), it was found that there was a significant order effect in which subjects who performed the patient task first had a faster overall reaction time $(737$ vs. $637 \mathrm{msec}) \quad[\mathrm{F}(1,36)=4.79$, $\mathrm{MSe}=42,293, \mathrm{p}<.05]$. This order effect did not, however, interact with the relation or target factors, and in all other respects, the analysis including order yielded the same results as the original analysis. Error rate was $1.88 \%$ for the fish stimuli and $4.58 \%$ for the W stimuli.

Postexperimental interviews with the spatial relation group revealed that none of the subjects gave an action relation interpretation to the $\mathrm{W}$ stimuli.

\section{Discussion}

This experiment illustrates the agent advantage effect. When the pictures contained a semantically interpretable transitive action relation (biting), the agent of the action was identified faster than the patient. When the pictures contained the same critical visual information but were interpreted in spatial relation terms, the agent advantage effect disappeared. This interaction effect increases our confidence that the agent advantage effect observed in the action relation condition results from the subjects' interpreting the pictures in terms of agent-patient relations. Had the spatial rather than the semantic properties contributed to the effect, one would have expected a parallel effect in the spatial relation condition. 
One question that arises is whether the faster latency to locate the agent reflects a difference in the relative speeds for extracting agent and patient information from the picture during encoding (cf. Olson \& Filby, 1972) or an agent-then-patient ordering of information in the mental representation of the event after encoding (Glucksberg et al., 1973). The purpose of the next experiment was to investigate these possibilities.

\section{EXPERIMENT 2}

In this experiment, a task was used in which subjects first viewed for $200 \mathrm{msec}$ a picture showing one animal biting another (e.g., a dog biting a cat) followed by a second picture in which either the agent or the patient had been replaced by a third animal (e.g., dog biting bird; bird biting cat). The subject's task in the agentmatch block of trials was to indicate whether the agent in the second picture was the same animal as the agent in the first picture. In a separate block of patient-match trials, the task was to indicate whether the patients in the two pictures were the same. The stimulus onset asynchrony (SOA) between the two stimulus pictures was systematically manipulated to determine the critical time $\left(\mathrm{SOA}_{\mathrm{c}}\right)$ needed to accurately perform the task. If the onset of the second picture occurred long enough before the relevant (agent or patient) information was extracted from the first picture, the match could not be made. Thus, the $\mathrm{SOA}_{\mathrm{c}}$ for the agent match provides an index of the time required to identify the agent in the first stimulus picture, and the $\mathrm{SOA}_{c}$ for the patientmatch task provides the analogous index for patient information extraction. A comparison of these two $\mathrm{SOA}_{\mathrm{c}} \mathrm{s}$ should indicate the relative speed with which information about the agent and patient can be encoded from the first stimulus picture.

After the agent-patient relations have been encoded, information is presumed to be placed in working memory for the comparison process. One possibility is that both the agent and the patient of the first picture are held in working memory before the comparison process can be accomplished and that this may be an ordered representation, with agent coming before patient. This is essentially the assumption made by Glucksberg et al. (1973) in their process model of performance in a picture-sentence verification task. Such ordering of agent-patient information would be revealed in faster reaction times when comparing the agents in the two pictures than when comparing the patients, since patient information in memory is available only after the agent information has been accessed.

This possibility was examined as follows. Once the $\mathrm{SOA}_{\mathrm{c}}$ had been determined for the agent-match task for a given subject, an additional 24 trials were given with the SOA set at $\mathrm{SOA}_{\mathrm{c}}$. This permitted determination of the subject's reaction time latency to complete the agent comparison under conditions favoring accurate perfor- mance. Reaction time for the patient comparison was similarly determined using the $\mathrm{SOA}_{\mathrm{c}}$ established in the patient-match trials. If agent-patient information is ordered in working memory, then the reaction times for the agent-match task should be faster than those for the patient-match task.

\section{Method}

Subjects. Sixteen paid subjects with normal or corrected-tonormal vision served as subjects in this experiment.

Materials. Stimulus materials consisted of black-on-white line drawings showing two different animals (bird, cat, or dog), one biting the other (as in Figure 2). All possible pairings of animals were used, with two different biting positions: one with the agent biting the leg of the patient (agent's head below patient's) and one with the agent biting the neck of the patient (agent's head above patient's). These variants were included to discourage subjects from adopting a position strategy to discover the agent or patient. Together with mirror images of the pictures, a basic deck of 24 stimulus pictures drawn on $3 \times 5$ in. cards was constructed.

Two copies were made of each stimulus card so that 24 pairings of pictures could be made. In half of these pairings, the target in the second picture was the same animal as the target in the first, and in half, the target in the second picture was different. The second picture always contained an animal not shown in the first; thus, the second picture never contained the same animals as the first with only the relations reversed.

Procedure. Subjects first saw one stimulus card for $200 \mathrm{msec}$, followed by a short, lighted blank interval before viewing a

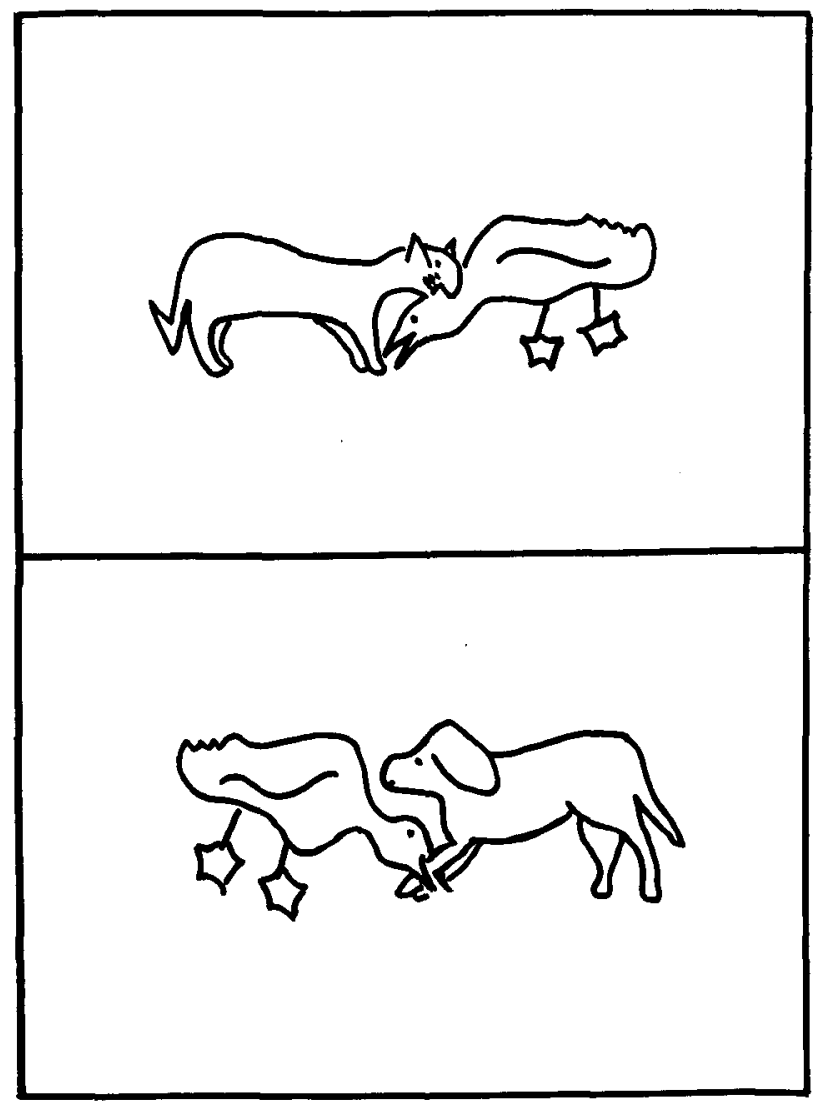

Figure 2. Sample stimuli from Experiment 2. 
second stimulus card for $200 \mathrm{msec}$. A pattern mask followed the second stimulus. In the agent-match condition, subjects were instructed to indicate whether the animal that was the agent ("giving the bite") in the first stimulus picture was also the agent in the second stimulus picture by pressing a reaction time panel (right for "same," left for "different"). In the patient-match condition, subjects had to compare the patients ("animal receiving the bite") in the two pictures.

Initially, the SOA was set at $230 \mathrm{msec}$. With each error, the SOA was increased by $30 \mathrm{msec}$ until the subject made five consecutive correct responses. With each subsequent error, the SOA was increased by $20 \mathrm{msec}$ until the subject had made eight consecutively correct responses. Finally, the SOA was further increased by $10 \mathrm{msec}$ after each error until the subject reached criterion of 10 consecutively correct responses. This final SOA was recorded as the $\mathrm{SOA}_{\mathrm{c}}$ for the particular task condition. Once criterion had been reached, subjects continued performing a further 24 trials with no change in SOA. Reaction times to respond during these 24 trials were recorded.

Half the subjects began with the agent-match task followed by the patient-match task, and half performed the tasks in the reverse order. There was a 5 -min break between the two tasks for all subjects.

Each task was introduced with a few warm-up examples at a slowed pace to ensure that subjects understood the task demands.

Design. This experiment consisted of two separate parts. Part 1 determined the $\mathrm{SOA}_{\mathrm{c}}$ for agent and patient matches, and Part 2 measured the response latencies to make the matches. Each part conformed to a simple one-factor (agent vs. patient) within-subjects design.

The basic sequence of 24 pairs of stimulus cards was recycled as long as necessary until the subject had reached the 24 th trial after completing the $10 \mathrm{SOA}_{\mathrm{c}}$ criterion trials. The sequencing of cards was pseudorandom, with the constraint that a given response (left or right panel press) never be required more than three consecutive times. Also, the agent or patient of the first stimulus was never repeated more than three consecutive times, and similarly for the second stimulus.

\section{Results}

The mean agent $\mathrm{SOA}_{\mathrm{c}}$ was significantly shorter $(389 \mathrm{msec})$ than the patient $\mathrm{SOA}_{\mathrm{c}}(474 \mathrm{msec})[\mathrm{t}(15)=$ $3.67, \mathrm{p}<.01]$. On the other hand, the analysis of variance of reaction times, with the factors being match (agent vs. patient) and response ("same" vs. "different") revealed that the reaction times in agent-match trials after criterion was reached were not significantly faster than were reaction times in patient-match trials $(1,111$ vs. $1,198 \mathrm{msec})[\mathrm{F}(1,15)=1.63, \mathrm{MSe}=74,998$, $\mathrm{p}>.05]$. There was, however, a significant response effect, with "same" $(1,111 \mathrm{msec})$ faster than "different" $(1,198 \mathrm{msec})$ (coincidently, reaction times similar to those for agent and patient) $[\mathrm{F}(1,15)=8.26$, MSe $=$ $14,790, p<.025]$. The Match by Response interaction was not significant $[F(1,15)=3.13, \quad M S e=16,465$, $p>.05]$. Overall error rate was $3.50 \%$.

\section{Discussion}

The main result of interest is the finding that the $\mathrm{SOA}_{\mathrm{c}}$ for agent is significantly shorter than the $\mathrm{SOA}_{\mathrm{c}}$ for patient, indicating that with the particular stimuli used, less processing time is required to identify the agent than to identify the patient. There are at least two possible explanations for the agent advantage effect obtained in this experiment. First, it may be that once the physical analysis of the picture is complete, encoding of agent-patient information occurs in a sequential and dependent manner in which encoding of patient information depends on prior analysis of agent information but not vice versa. A second possible explanation is that agent and patient information can be analyzed independently of each other but that agent information is more quickly and efficiently encoded. This view is consistent with Olson and Filby's (1972) contention that it is easier to "read a picture in the active voice." The data from the present experiment do not allow us to decide between these two views, but they do support the view that there is an agent-patient difference during encoding.

The reaction time data can be interpreted as evidence against an ordering of agent-patient information in working memory, since no significant difference between agent- and patient-match reaction times was found. Since reaction time was measured from onset of the second picture, one might have expected at least some difference reflecting an agent advantage effect for encoding the second picture. No significant reaction time difference appeared, however, indicating an absence of agent-patient differences after the first picture was encoded (some caution is in order regarding this negative conclusion, since the difference obtained was of the same magnitude as the significant $\mathrm{SOA}_{\mathbf{c}}$ difference). This result differs from the picture-sentence verification studies cited earlier that tend to show that the semantic representation of a picture orders agent information before patient information. It may be that such ordering exists when the task includes sentence processing (e.g., Wannemacher, 1976 , p. 50 , on the encoding format for pictures), since the language favors an agent-patient order of mention, but that such ordering does not necessarily exist in the case of picture processing, as indicated in this experiment.

The finding that reaction time for "same" responses was significantly faster than reaction time for "different" responses is consistent with the view that some serial processing is involved in the comparison task (cf. Glucksberg et al., 1973). Positive matches will, on average, be made faster than negative matches if the process is self-terminating.

In summary, the critical SOA data suggest that there is an agent-patient difference during encoding, but the reaction time data do not suggest a difference in stages after encoding. Experiment 3 takes up the issue of whether the encoding effect reflects a sequential and dependent relation regarding agent and patient processing or whether such processing proceeds independently for agents and patients but more efficiently for agents. 


\section{EXPERIMENT 3}

The previous experiment indicated an agent-patient effect during encoding. This raises the question of what exactly happens during encoding that results in an advantage for processing agent information. Two possibilities are explored here. As mentioned earlier, it could be that the analysis of the picture involves a dependent relationship in which the encoding of patient information depends on agent encoding but not vice versa, regardless of the task demands. A second possibility is that while agent and patient information can be encoded independently of each other, the agent is encoded faster than the patient. This might occur, perhaps, because the search for the defining characteristics of the agent may be better directed and hence faster than the search for the defining characteristics of the patient. For example, "biting" necessarily involves the agent's mouth, a highly specified part of its body in the pictures used, but receiving a bite could involve any part of the patient's body, since the attacker can as easily bite from behind as from above, below, or the side.

The purpose of the present experiment was to assess these two possibilities. Subjects briefly viewed a stimulus similar to the one in Figure 3 in which one fish was drawn in red outline and the others in black. The subject's task in one block of trials was to indicate whether the red fish was the agent and in another block whether it was the patient. The presence of distracting pairs of fish was intended to heighten the value of color as a clue about where to begin analysis of the target pair of fish.

In each block of trials, the correct response was negative half the time. In half of these negative trials,

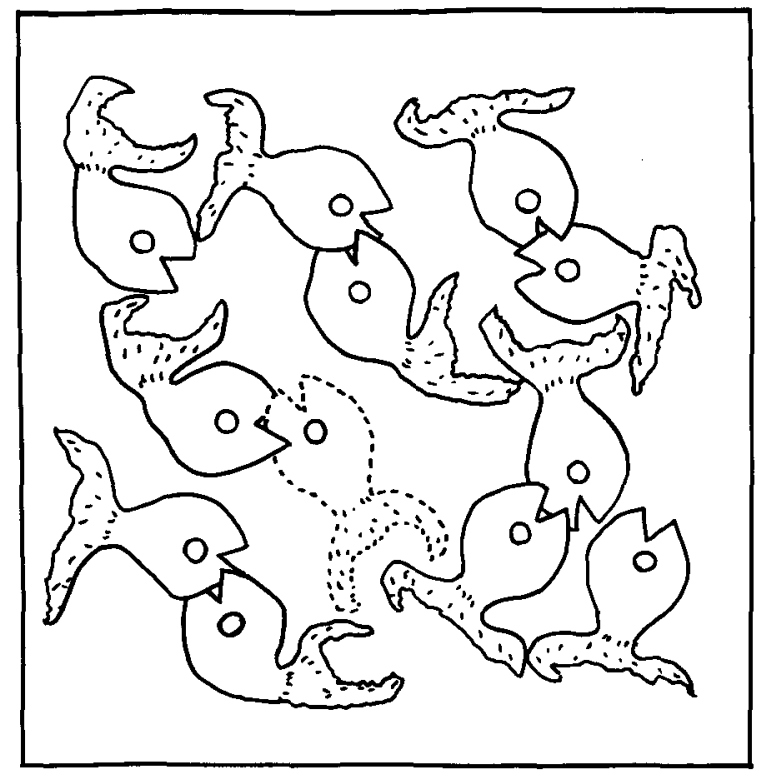

Figure 3. Sample stimulus from Experiment 3. All fish were drawn in solid black outline except one, which was drawn in red solid outline (here shown in broken outline). however, the red fish was neither agent nor patient (no biting). Inclusion of such no-action trials permitted determination of whether patient encoding depends upon agent encoding (the sequential dependency hypothesis) or whether agent encoding is simply faster than patient encoding (the independence hypothesis).

The sequential dependence hypothesis. Under this hypothesis, encoding the patient depends upon first encoding the agent, but not vice versa. Thus, in the agent-judgment condition, in which subjects search for the agent, the subjects are presumed to examine an element for "agentness" and accept or reject it regardless of the presence of a patient element. It is predicted, therefore, that reaction times when the red fish is not the agent will be similar for both action pictures (in which the black fish is the agent) and no-action pictures (in which neither fish is agent).

In the patient-judgment condition, however, the situation is somewhat more complex, because encoding the patient element is presumed to depend on prior encoding of the agent. Therefore, on negative trials containing an agent (action picture), responses should be quicker than on trials not containing an agent (noaction pictures). Thus the sequential dependence hypothesis predicts an interaction effect, with different reaction times in the patient-judgment condition for the two types of negative trials but no such difference in the agent-judgment condition.

The independence hypothesis. Under this hypothesis, the viewer is presumed to be able to encode either agent or patient information independently, but to be faster with agent information. Here, one expects that while patient judgments should produce slower responses than agent judgments, there should be equally fast responses on the two types of negative patient-judgment trials, since encoding of the patient does not depend on the presence of an agent.

\section{Method}

Subjects. Sixteen paid subjects with normal or corrected-tonormal vision served in this experiment.

Materials. Stimulus materials consisted of line drawings of 12 fish, as in Figure 3. All the fish were drawn in black outline except for one drawn in red; the pair with the red fish was designated the target pair. The agent-patient characteristics and the physical characteristics were varied as described in Experiment 1 . The location of the target pair within the whole array was varied in a counterbalanced fashion.

Procedure. Subjects viewed the stimulus picture (wallprojected color slides) for $1,000 \mathrm{msec}$. In one block of trials, they had to indicate whether the red fish was agent, and in a different block, they had to indicate whether the red fish was patient. Each block consisted of 53 trials: The first 5 were buffer trials and not counted in the final analyses; 24 were affirmative trials, 12 were negative action trials, and 12 were negative no-action trials.

\section{Results and Discussion}

Preliminary analysis of the data showed no significant differences in the pattern of results for those subjects performing the agent task first compared with those 


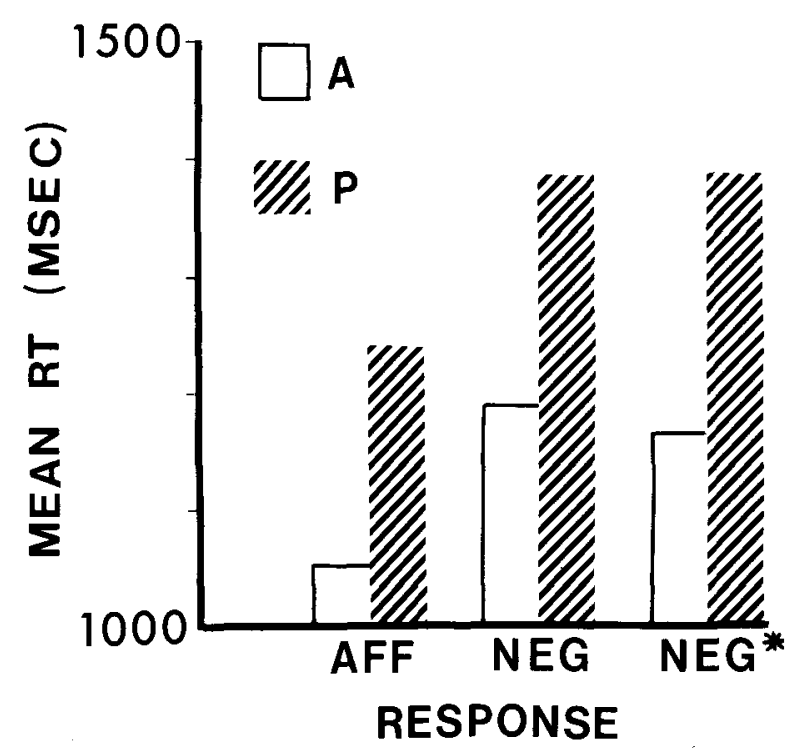

Figure 4. Reaction times to make agent and patient judgments on affirmative (AFF) and negative (NEG) trials. On NEG* trials, no biting action was depicted.

performing the patient task first (all Fs with this factor $<1$ ). Consequently, in the analysis reported below, data are collapsed over order of presentation.

Figure 4 shows the mean reaction times for responding affirmatively and negatively in the agent- and patientjudgment blocks. Analysis of variance revealed a significant agent-patient difference $(1,137$ vs. $1,347 \mathrm{msec})$ $[F(1,15)=30.73, \mathrm{MSe}=34,641, \mathrm{p}<.001]$, indicating overall faster responding in the agent-judgment condition. The results also revealed a significant trial-type difference (affirmative trials $=1,148 \mathrm{msec}$; negative action trials $=1,293 \mathrm{msec}$; negative no-action trials = $1,286 \mathrm{msec})[\mathrm{F}(2,30)=18.73, \mathrm{MSe}=11,287, \mathrm{p}<.001]$, indicating that affirmative responses were faster than negative responses, but that negative action and no-action responses did not differ. The interaction effect was not significant $[F(2,30)=.39, \mathrm{MSe}=5,654, \mathrm{p}>.25]$. Overall error rate was $5.33 \%$.

As can be seen from Figure 4, the pattern of results conformed to the prediction of the independence hypothesis: overall faster agent processing and similar reaction times for agent judgments and patient judgments across the action and no-action conditions. In general, one can see that the presence or absence of an agent had no effect on the response times, supporting the hypothesis that encoding the patient element occurs independently of encoding the agent.

\section{GENERAL DISCUSSION}

The three experiments reported here present evidence of an "agent advantage effect" in picture processing. Experiment 1 demonstrated the effect in a paradigm in which reaction time to manually indicate the location of a target was faster for agents than for patients. In
Experiment 2, in which subjects were required to match the agents or patients of two successively presented pictures, the critical interval between pictures required for errorless responding was shorter for agent matches. On the other hand, reaction times were the same for agent and patient matches when a sufficient interval was provided for errorless responding. This indicated that the agent advantage effect reflected a difference in agent and patient processing at the encoding stage. Finally, Experiment 3 demonstrated the agent advantage effect again in a directed attention paradigm. The results indicated that encoding of agents and patients occurred independently but faster for agents.

Because the present study used only the action "biting," a crucial question about these results is whether they are generalizable to other visually depicted actions. An argument against such an expectation follows from the suggestion made earlier that, in the pictures used here, the agent may be better specified than the patient. This notion was not directly tested in the present series, but if it is correct, then one must inquire whether there are other visually depictable actions that do not specify the agent better than the patient or that perhaps even specify the patient better.

This notion of agent or patient specificity is reminiscent of an observation by Garvey and Caramazza (1974) regarding linguistic biases among certain verbs. They demonstrated that certain verbs may "point" to the subject of the sentence whereas others "point" to the object as the best candidate for the grammatical subject of the next clause. For example, in the sentence fragment, "The mother punished her daughter because she ...," the ambiguous pronoun "she" is more often assigned to the object of the main clause, "daughter," in a sentence-completion task. Garvey and Caramazza discuss the reasons for this in terms of the way the verbs impute causes of the event or situation to some factor associated primarily with the subject- or object-noun phrase antecedent. A verb can thus be seen to serve as a label that specifies a set of relations among the participants of the event described in the sentence, and it also serves to highlight specific aspects of these relations in particular ways. In the present research, the verb "bite" was used to designate the action in the pictures; perhaps the agent advantage effect reflects the way that particular label specifies the relations between participants in the "bite" event.

A final issue that deserves consideration is the possible relation between the agent advantage effect in the processing of visual displays and agent-patient ordering effects in language structure. The agent-patient order of mention is far more common across the world's languages than is the reverse (Steele, 1978). One avenue for speculation of why this is so may be suggested by the present results (see also Segalowitz \& Hansson, 1979). Perhaps it is indeed generally true that the encoding of nonlinguistic agent information occurs more 
rapidly than the encoding of patient information. If so, then agent information would generally be available to working memory first and thus facilitate an agentpatient order of mention in sentence formation. Future research on the generalizability of the agent advantage effect to other visually depicted actions and the study of the mechanism underlying the effect may thus contribute to an understanding of the subject-object order of mention noted in the majority of the world's languages.

\section{REFERENCES}

BAldie, B. The acquisition of the passive voice. Journal of Child Language, 1976, 3, 331-348.

Bever, T. The cognitive basis for linguistic structures. In J. R. Hayes (Ed.), Cognition and the development of language. New York: Wiley, 1970.

Clark, H., Carpenter, P., \& Just, M. On the meeting of semantics and perception. In W. G. Chase (Ed.), Visual information processing. New York: Academic Press, 1973.

Garvey, C., \& Caramazza, A. Implicit causality in verbs. Linguistic Inquiry, 1974, 5, 459-464.

Glucksberg, S., Trabasso, T., \& Wald, J. Linguistic structures and mental operations. Cognitive Psychology, 1973, 5, 338-370.

GreEnBerg, J. Some universals of grammar with particular reference to the order of meaningful elements. In J. Greenberg (Ed.), Universals of language. Cambridge, Mass: M.I.T. Press, 1963.
KeEnan, E. Towards a universal definition of "subject." In C. Li (Ed.), Subject and topic. New York: Academic Press, 1976.

Olson, D., \& Filby, N. On the comprehension of active and passive sentences. Cognitive Psychology, 1972, 3, 361-381.

Pullum, J. Word order universals and grammatical relations. In P. Cole \& J. Sadock (Eds.), Syntax and semantics (Vol. 8): Grammatical relations. New York: Academic Press, 1977.

Segalowitz, N., \& Galang, R. Agent-patient word order preference in the acquisition of Tagalog. Journal of Child Language, $1978,5,47-64$

Segalowitz, N., \& Hansson, P. Hemispheric functions in the processing of agent-patient information. Brain and Language, $1979,8,51-61$.

Slobin, D. Cognitive prerequisites for the development of grammar. In C. A. Ferguson \& D. Slobin (Eds.), Studies of child language development. New York: Holt, Rinehart \& Winston, 1973.

Ste ele, S. Word order variation: A typological study. In J. Greenberg (Ed.), Universals of human language (Vol. 4): Syntax. Stanford, Calif: Stanford University Press, 1978.

Strohner, H., \& Nelson, K. The young child's development of sentence comprehension: Influence of event probability, nonverbal context, syntactic form and strategies. Child Development, 1974, 43, 567-576.

Wannemacher, J. Processing strategies in picture-sentence verification tasks. Memory \& Cognition, 1974, 2, 554-560.

Wannemacher, J. Processing strategies in sentence comprehension. Memory \& Cognition, 1976, 4, 48-52.

(Received for publication August 21, 1981; revision accepted March 18, 1982.) 